\title{
Further extension of Voigt function and its properties
}

\author{
Nabiullah Khan ${ }^{1}$, Mohd Ghayasuddin², Waseem A. Khan ${ }^{3}$, Thabet Abdeljawad ${ }^{4,5,6}$ and Kottakkaran
} Sooppy Nisar $^{7 *}$ (D)

\section{"Correspondence:}

n.sooppy@psau.edu.sa

${ }^{7}$ Department of Mathematics, College of Arts and Sciences, Prince Sattam bin Abdulaziz University, Wadi Aldawasir, Kingdom of Saudi Arabia

Full list of author information is available at the end of the article

\section{Springer}

\begin{abstract}
In this paper, by using the confluent hypergeometric function of the first kind, we propose a further extension of the Voigt function and obtain its useful properties as (for example) explicit representation and partly bilateral and partly unilateral representation. By means of the present representations, we derive several (presumably new) generating functions which are partly bilateral and partly unilateral. Some interesting recurrence relations of the Voigt function introduced here are also indicated.
\end{abstract}

MSC: Primary 33C15; 33C70; 33E20; secondary 85A99

Keywords: Voigt function; Kampé de Fériet function; Srivastava and Daoust function

\section{Introduction and preliminaries}

The familiar Voigt functions $K(x, y)$ and $L(x, y)$ were introduced and investigated by Voigt in 1899. Mainly due to their applications in diverse research areas, such as astrophysical spectroscopy, neutrons physics, statistical communication theory, and plasma physics, the Voigt functions and their various generations have been intensively and extensively investigated by many authors. For a review of the (unification) generalizations of Voigt functions introduced from time to time, see Srivastava and Miller [17], Klusch [6], Srivastava and Chen [15], Gupta et al. [4], Pathan and Shahwan [10], Goyal and Mukherjee [2], Pathan et al. [9], Srivastava et al. [18], Pathan et al. [8], Gupta and Gupta [3], Khan et al. [5], etc.

We recall here the generalized Voigt function defined by Srivastava et al. [18, p. 53, Eq. (1.27)] needed for the present investigation:

$$
\begin{gathered}
\Omega_{\eta, v, \lambda}^{\mu}[x, y, z]=\sqrt{\frac{x}{2}} \int_{0}^{\infty} t^{\eta} e^{-y t-z t^{2}} J_{v, \lambda}^{\mu}(x t) d t \\
\left(x, y, z, \mu \in \Re^{+} ; \Re(\eta+v+2 \lambda)>-1\right),
\end{gathered}
$$

where $J_{v, \lambda}^{\mu}(z)$ is the well-known Bessel-Maitland function defined as follows (see [7]):

$$
J_{v, \lambda}^{\mu}(z)=\sum_{m=0}^{\infty} \frac{(-1)^{m}(z / 2)^{v+2 \lambda+2 m}}{\Gamma(\lambda+m+1) \Gamma(v+\lambda+\mu m+1)} .
$$

(c) The Author(s) 2020. This article is licensed under a Creative Commons Attribution 4.0 International License, which permits use, sharing, adaptation, distribution and reproduction in any medium or format, as long as you give appropriate credit to the original author(s) and the source, provide a link to the Creative Commons licence, and indicate if changes were made. The images or other third party material in this article are included in the article's Creative Commons licence, unless indicated otherwise in a credit line to the material. If material is not included in the article's Creative Commons licence and your intended use is not permitted by statutory regulation or exceeds the permitted use, you will need to obtain permission directly from the copyright holder. To view a copy of this licence, visit http://creativecommons.org/licenses/by/4.0/. 
Note that on setting $z=\frac{1}{4}$ in equation (1), the generalized Voigt function defined by Srivastava and Chen [15] can be retrieved. If we set $\lambda=0$ and $\mu=1$ in equation (1), then it reduces to the generalized Voigt function given by Klusch [6], which, on taking $z=\frac{1}{4}$, further reduces to the Voigt function introduced by Srivastava and Miller [17].

The paper aims at presenting a new extension of the Voigt function defined by equation (1) in a slightly modified form by involving the confluent hypergeometric function as follows:

$$
\begin{gathered}
\Lambda_{\eta, v, \lambda}^{\mu, \alpha, \beta}[x, y, z]=\sqrt{\frac{x}{2}} \int_{0}^{\infty} t^{\eta} e^{-z t^{2}}{ }_{1} F_{1}(\alpha ; \beta ;-y t) J_{v, \lambda}^{\mu}(x t) d t \\
\left(x, y, z, \mu, \alpha, \beta \in \Re^{+} ; \Re(\eta+v+2 \lambda)>-1\right),
\end{gathered}
$$

where ${ }_{1} F_{1}(a ; b ; z)$ is the confluent hypergeometric function defined as follows (see [16, p. 36, Eq. (3)]):

$$
{ }_{1} F_{1}(a ; b ; z)=\sum_{n=0}^{\infty} \frac{(a)_{n}}{(b)_{n}} \frac{z^{n}}{n !} .
$$

If we set $a=b$, then equation (4) reduces to

$$
{ }_{1} F_{1}(a ; a ; z)=e^{z}
$$

For $\alpha=\beta$, our extended Voigt function defined by equation (3) reduces to the Voigt function defined by Srivastava et al. [18, p. 53, Eq. (2.7)].

\section{A series representation}

In order to derive the explicit representation of our extended Voigt function in terms of the familiar special functions of the mathematical physics, we make use of the series representation of the confluent hypergeometric function and Bessel-Maitland function defined by equation (4) and equation (2), respectively. Reversing the order of summation and integration (under the given conditions), we get

$$
\begin{aligned}
\Lambda_{\eta, v, \lambda}^{\mu, \alpha, \beta}[x, y, z]= & \left(\frac{x}{2}\right)^{\nu+2 \lambda+\frac{1}{2}} \sum_{m, n=0}^{\infty} \frac{(-1)^{m}(\alpha)_{n}(x / 2)^{2 m}(-y)^{n}}{\Gamma(\lambda+m+1) \Gamma(v+\lambda+\mu m+1)(\beta)_{n} n !} \\
& \times \int_{0}^{\infty} t^{\eta+\nu+2 \lambda+2 m+n} e^{-z t^{2}} d t .
\end{aligned}
$$

Applying the following integral, which is easily deducible from the familiar Euler gamma function,

$$
\begin{gathered}
\int_{0}^{\infty} t^{\lambda} e^{-z t^{2}} d t=\frac{1}{2} \Gamma\left(\frac{\lambda+1}{2}\right) z^{-}\left(\frac{\lambda+1}{2}\right) \\
(\mathfrak{R}(z)>0, \mathfrak{R}(\lambda)>-1),
\end{gathered}
$$

to the integral in equation (6), we obtain

$$
\Lambda_{\eta, v, \lambda}^{\mu, \alpha, \beta}[x, y, z]=\frac{z^{-\left(\frac{\eta+v+2 \lambda+1}{2}\right)} x^{\nu+2 \lambda+1 / 2}}{2^{v+2 \lambda+3 / 2}}
$$




$$
\begin{aligned}
\times \sum_{m, n=0}^{\infty} \frac{(\alpha)_{n}\left(-\frac{x^{2}}{4 z}\right)^{m}\left(-\frac{y}{\sqrt{z}}\right)^{n} \Gamma\left(\frac{\eta+v+2 \lambda+1+2 m+n}{2}\right)}{(\beta)_{n} \Gamma(\lambda+m+1) \Gamma(v+\lambda+\mu m+1) n !} \\
\left(x, y, z, \mu, \alpha, \beta \in \mathfrak{R}^{+} ; \Re(\eta+v+2 \lambda)>-1\right) .
\end{aligned}
$$

On splitting the $n$-series into even and odd terms, we obtain

$$
\begin{aligned}
\Lambda_{\eta, v, \lambda}^{\mu, \alpha, \beta}[x, y, z]= & \frac{z^{-\left(\frac{\eta+v+2 \lambda+1}{2}\right)} x^{v+2 \lambda+1 / 2}}{2^{v+2 \lambda+3 / 2}}\left[\frac{(\alpha)_{2 n}\left(-\frac{x^{2}}{4 z}\right)^{m}\left(\frac{y^{2}}{4 z}\right)^{n} \Gamma\left(\frac{\eta+v+2 \lambda+1}{2}+m+n\right)}{(\beta)_{2 n}\left(\frac{1}{2}\right)_{n} \Gamma(\lambda+m+1) \Gamma(v+\lambda+\mu m+1) n !}\right. \\
& \left.-\frac{y}{\sqrt{z}} \sum_{m, n=0}^{\infty} \frac{(\alpha)_{2 n+1}\left(-\frac{x^{2}}{4 z}\right)^{m}\left(\frac{y^{2}}{4 z}\right)^{n} \Gamma\left(\frac{\eta+v+2 \lambda+2}{2}+m+n\right)}{(\beta)_{2 n+1}\left(\frac{3}{2}\right)_{n} \Gamma(\lambda+m+1) \Gamma(v+\lambda+\mu m+1) n !}\right] .
\end{aligned}
$$

For $\alpha=\beta$, equation (9) reduces to the explicit representation of Voigt function defined by Srivastava et al. [18, p. 55, Eq. (2.4)].

On using the definition of Kampé de Fériet function $F_{s: t ; u}^{p: q ; r}$ (see [16, p. 63]) in equation (9), we arrive at the following explicit representation of our extended Voigt function $\Lambda_{\eta, v, \lambda}^{\mu, \alpha, \beta}$ :

$$
\begin{aligned}
& \Lambda_{\eta, v, \lambda}^{\mu, \alpha, \beta}[x, y, z] \\
& =\frac{x^{\nu+2 \lambda+\frac{1}{2}}}{2^{\nu+2 \lambda+\frac{3}{2}} z^{p} \Gamma(\lambda+1) \Gamma(\nu+\lambda+1)} \\
& \times\left\{\Gamma(P) F_{0: \mu+1 ; 3}^{1: 1 ; 2}\left[\begin{array}{cc}
P: 1 ; \Delta(2 ; \alpha+1) ; & -\frac{x^{2}}{4 z \mu^{\mu}}, \frac{y^{2}}{4 z} \\
-: \lambda+1, \Delta(\mu ; \nu+\lambda+1) ; \Delta(2 ; \beta), \frac{1}{2} ; &
\end{array}\right]\right. \\
& \left.-\frac{\alpha y}{\beta \sqrt{z}} \Gamma\left(p+\frac{1}{2}\right) F_{0: \mu+1 ; 3}^{1: 1 ; 2}\left[\begin{array}{cc}
P+\frac{1}{2}: 1 ; \Delta(2 ; \alpha+1) ; \\
-: \lambda+1, \Delta(\mu ; \nu+\lambda+1) ; \Delta(2 ; \beta+1), \frac{3}{2} ; & -\frac{x^{2}}{4 z \mu^{\mu}}, \frac{y^{2}}{4 z}
\end{array}\right]\right\} \\
& \left(x, y, z, \mu, \alpha, \beta \in \mathfrak{R}^{+} ; \Re(\lambda)>-1, \Re(\nu+\lambda)>-1, \Re(P)>0\right) \text {, }
\end{aligned}
$$

where $P=\frac{\eta+v+2 \lambda+1}{2}$ and $\Delta(m ; a)$ abbreviates the array of $m$ parameters $\frac{a}{m}, \frac{a+1}{m}, \ldots, \frac{a+m+1}{m}$, $m \geq 1$.

For the case $\beta=\alpha$, equation (10) reduces to the representation defined by Srivastava et al. [18] in a slightly modified form (i.e., in terms of the parameters $\mu$ and $\lambda$ ):

$$
\begin{aligned}
& \Lambda_{\eta, v, \lambda}^{\mu, \alpha, \alpha}[x, y, z] \\
& =\Omega_{\eta, v, \lambda}^{\mu}[x, y, z] \\
& =\frac{x^{\nu+2 \lambda+\frac{1}{2}}}{2^{v+2 \lambda+\frac{3}{2}} z^{p} \Gamma(\lambda+1) \Gamma(v+\lambda+1)} \\
& \times\left\{\Gamma(P) F_{0: \mu+1 ; 1}^{1: 10}\left[\begin{array}{cc}
P: 1 ;-; & -\frac{x^{2}}{4 z \mu^{\mu}}, \frac{y^{2}}{4 z} \\
-: \lambda+1, \Delta(\mu ; \nu+\lambda+1) ; \frac{1}{2} ; &
\end{array}\right]\right. \\
& \left.-\frac{y}{\sqrt{z}} \Gamma\left(p+\frac{1}{2}\right) F_{0: \mu+1 ; 1}^{1: 1 ; 0}\left[\begin{array}{cc}
P+\frac{1}{2}: 1 ;-; & -\frac{x^{2}}{4 z \mu^{\mu}}, \frac{y^{2}}{4 z} \\
-: \lambda+1, \Delta(\mu ; \nu+\lambda+1) ; \frac{3}{2} ; &
\end{array}\right]\right\}
\end{aligned}
$$




$$
\left(x, y, z, \mu \in \mathfrak{R}^{+} ; \mathfrak{R}(\lambda)>-1, \mathfrak{R}(v+\lambda)>-1, \mathfrak{R}(P)>0\right) .
$$

On setting $y=0$ in equation (10) and equation (11), respectively, we get the following (presumably new) interesting result:

$$
\begin{aligned}
& \Lambda_{\eta, v, \lambda}^{\mu, \alpha, \beta}[x, 0, z] \\
& =\Omega_{\eta, v, \lambda}^{\mu}[x, 0, z] \\
& =\frac{x^{\nu+2 \lambda+\frac{1}{2}} \Gamma(P)}{2^{v+2 \lambda+\frac{3}{2}} z^{p} \Gamma(\lambda+1) \Gamma(v+\lambda+1)} \\
& \quad \times{ }_{2} F_{\mu+1}\left[\begin{array}{cr}
P: 1 ; & \left.-\frac{x^{2}}{4 z \mu^{\mu}}\right] \\
\lambda+1, \Delta(\mu ; v+\lambda+1) ; &
\end{array}\right] \\
& \quad\left(x, z, \mu \in \Re^{+} ; \Re(\lambda)>-1, \Re(v+\lambda)>-1, \Re(P)>0\right),
\end{aligned}
$$

where ${ }_{p} F_{q}$ is the generalized hypergeometric function (see [12, p. 73]).

Thus, we obtain

$$
\Lambda_{\eta, v, \lambda}^{\mu, \alpha, \beta}[x, 0, z]=\Omega_{\eta, v, \lambda}^{\mu}[x, 0, z]
$$

where $\Omega_{\eta, v, \lambda}^{\mu}$ corresponds to the generalized Voigt function given in equation (1) (see, for details, [18]).

\section{Partly bilateral and partly unilateral representation of $\Lambda_{\eta, v, \lambda}^{\mu, \alpha, \beta}$}

We begin by recalling here the following known result (see [14, p. 8, Eq. (1.3)]):

$$
\exp \left[s+t-\frac{x t}{s}\right]=\sum_{m=-\infty}^{\infty} \sum_{p=0}^{\infty} \frac{s^{m}}{m !} \frac{t^{p}}{p !}{ }_{1} F_{1}[-p ; m+1 ; x]
$$

where ${ }_{1} F_{1}$ is the confluent hypergeometric function defined by equation (4).

The replacement of $s, t$, and $x$ by $s \xi^{2}, t \xi^{2}$, and $x \xi^{2}$, respectively, and further multiplying both sides of the resulting identity by $\xi^{\eta} e^{-z \xi^{2}}{ }_{1} F_{1}(\alpha ; \beta ;-w \xi) J_{v, \lambda}^{\mu}(q \xi)$ and integrating both sides of the last resulting identity with respect to $\xi$ from 0 to $\infty$, and interchanging the summations and integration gives

$$
\begin{aligned}
& \int_{0}^{\infty} \xi^{\eta} \exp \left[-\left(z-s-t+\frac{x t}{s}\right) \xi^{2}\right]{ }_{1} F_{1}(\alpha ; \beta ;-w \xi) J_{v, \lambda}^{\mu}(q \xi) d \xi \\
&= \sum_{m=-\infty}^{\infty} \sum_{p=0}^{\infty} \frac{s^{m}}{m !} \frac{t^{p}}{p !} \int_{0}^{\infty} \xi^{\eta+2 m+2 p} e^{-z \xi^{2}}{ }_{1} F_{1}(\alpha ; \beta ;-w \xi) \\
& \quad \times J_{v, \lambda}^{\mu}(q \xi)_{1} F_{1}\left[-p ; m+1 ; x \xi^{2}\right] d \xi
\end{aligned}
$$

On comparing equation (15) and equation (3), we get

$$
\Lambda_{\eta, v, \lambda}^{\mu, \alpha, \beta}\left[q, w, z-s-t+\frac{x t}{s}\right]
$$




$$
\begin{aligned}
= & \sqrt{\frac{q}{2}} \sum_{m=-\infty}^{\infty} \sum_{p=0}^{\infty} \frac{s^{m}}{m !} \frac{t^{p}}{p !} \int_{0}^{\infty} \xi^{\eta+2 m+2 p} e^{-z \xi^{2}}{ }_{1} F_{1}(\alpha ; \beta ;-w \xi) \\
& \times J_{v, \lambda}^{\mu}(q \xi)_{1} F_{1}\left[-p ; m+1 ; x \xi^{2}\right] d \xi
\end{aligned}
$$

Expanding the confluent hypergeometric function ${ }_{1} F_{1}(\alpha ; \beta ;-w \xi)$ and Bessel-Maitland function $J_{v, \lambda}^{\mu}(q \xi)$ in equation (16) in their defining series, interchanging the integration and summations, and finally integrating the involved integral with the help of the following known integral formula (see [1, p. 337, Eq. (9)]):

$$
\begin{aligned}
& \int_{0}^{\infty} x^{s-1} e^{-\alpha x^{2}}{ }_{1} F_{1}\left(a ; b ; \beta x^{2}\right) d x=\frac{1}{2} \alpha^{-s / 2} \Gamma\left(\frac{s}{2}\right){ }_{2} F_{1}\left(a, \frac{s}{2} ; b ; \frac{\beta}{\alpha}\right) \\
& (\Re(\alpha)>\max \{0, \Re(\beta)\} ; \Re(s)>0),
\end{aligned}
$$

we obtain

$$
\begin{aligned}
\Lambda_{\eta, v, \lambda}^{\mu, \alpha, \beta} & {\left[q, w, z-s-t+\frac{x t}{s}\right] } \\
= & \frac{q^{\nu+2 \lambda+\frac{1}{2}}}{2^{v+2 \lambda+\frac{3}{2}} z^{P}} \sum_{m=-\infty}^{\infty} \sum_{p=0}^{\infty} \frac{\left(\frac{s}{z}\right)^{m}}{m !} \frac{\left(\frac{t}{z}\right)^{p}}{p !} \\
& \times \sum_{i, j=0}^{\infty} \frac{(\alpha)_{i}\left(\frac{-w}{\sqrt{z}}\right)^{i}\left(\frac{-q^{2}}{4 z}\right)^{j} \Gamma\left(P+m+p+j+\frac{i}{2}\right)}{(\beta)_{i} \Gamma(\lambda+j+1) \Gamma(v+\lambda+\mu j+1) i !} \\
& \times{ }_{2} F_{1}\left(-p, P+m+p+j+\frac{i}{2} ; m+1 ; \frac{x}{z}\right),
\end{aligned}
$$

where $P=\frac{\eta+v+2 \lambda+1}{2}$. For $\alpha=\beta$, equation (18) reduces to the known result of Srivastava et al. [18, p. 59, Eq. (3.5)].

Now expanding the hypergeometric function ${ }_{2} F_{1}$ in its defining series, separation of $i$ series into its even and odd terms, and a little simplification leads us to

$$
\begin{aligned}
& \Lambda_{\eta, v, \lambda}^{\mu, \alpha, \beta}\left[q, w, z-s-t+\frac{x t}{s}\right] \\
& =\frac{q^{v+2 \lambda+\frac{1}{2}}}{2^{v+2 \lambda+\frac{3}{2}} z^{p} \Gamma(\lambda+1) \Gamma(v+\lambda+1)} \sum_{m=-\infty}^{\infty} \sum_{p=0}^{\infty} \frac{\left(\frac{s}{z}\right)^{m}}{m !} \frac{\left(\frac{t}{z}\right)^{p}}{p !} \\
& \times\left\{\Gamma(P+m+p) F_{0: 2 ; 2 ; 1}^{1: 1 ; 1 ; 1}\left[\begin{array}{cc}
(P+m+p: 1,1,1):(\alpha, 2) ;(1,1) ;(-p, 1) ; \\
-:(\beta, 2),\left(\frac{1}{2}, 1\right) ;(\lambda+1,1),(v+\lambda+1, \mu) ;(m+1,1)
\end{array} \quad \frac{w^{2}}{4 z},-\frac{q^{2}}{4 z}, \frac{x}{z}\right]\right. \\
& -\frac{w}{\sqrt{z}} \frac{\alpha}{\beta} \Gamma\left(P+p+m+\frac{1}{2}\right) \\
& \left.\times F_{0: 2 ; 2 ; 1}^{1: 1 ; 1 ; 1}\left[\begin{array}{cc}
\left(P+m+p+\frac{1}{2}: 1,1,1\right):(\alpha+1,2) ;(1,1) ;(-p, 1) ; & \frac{w^{2}}{4 z},-\frac{q^{2}}{4 z}, \frac{x}{z} \\
-:(\beta+1,1),\left(\frac{3}{2}, 1\right) ;(\lambda+1,1),(v+\lambda+1, \mu) ;(m+1,1) ; &
\end{array}\right]\right\} \\
& \left(x, y, z, \mu, \alpha, \beta \in \mathfrak{R}^{+} ; \mathfrak{R}(\lambda)>-1, \mathfrak{\Re}(\nu)>0, \Re(P)>0\right) \text {, }
\end{aligned}
$$

where $F_{e: f ; g ; h}^{a: b ; c ;}$ is the well-known Srivastava and Daoust function [16, p. 64]. 
On setting $\alpha=\beta$ in equation (19), and after some simplification, we get the representation (partly bilateral and partly unilateral) of the Voigt function given in $[18$, p. 59, Eq. (3.6)].

\section{Generating functions}

In this section, we give a set of (presumably) new generating functions which are partly bilateral and partly unilateral.

A generating relation between the Kampé de Fériet function and Srivastava and Daoust function can be obtained by expanding the L.H.S. of equation (19) with the help of equation (10). We have indeed

$$
\begin{aligned}
& \left(\frac{z}{Z}\right)^{P}\left\{\Gamma(P) F_{0: \mu+1 ; 3}^{1: 1 ; 2}\left[\begin{array}{cc}
P: 1 ; \Delta(2 ; \alpha) ; & -\frac{q^{2}}{4 Z \mu^{\mu}}, \frac{w^{2}}{4 Z} \\
-: \lambda+1, \Delta(\mu ; \nu+\lambda+1) ; \Delta(2 ; \beta), \frac{1}{2} ;
\end{array}\right]\right. \\
& \left.-\frac{\alpha w}{\beta \sqrt{Z}} \Gamma\left(P+\frac{1}{2}\right) F_{0: \mu+1 ; 3}^{1: 1 ; 2}\left[\begin{array}{cc}
P+\frac{1}{2}: 1 ; \Delta(2 ; \alpha+1) ; & -\frac{q^{2}}{4 Z \mu^{\mu}}, \frac{w^{2}}{4 Z}
\end{array}\right]\right\} \\
& =\sum_{m=-\infty}^{\infty} \sum_{p=0}^{\infty} \frac{\left(\frac{s}{z}\right)^{m}}{m !} \frac{\left(\frac{t}{z}\right)^{p}}{p !} \\
& \times\left\{\Gamma(P+m+p) F_{0: 2 ; 2 ; 1}^{1: 1 ; 1 ;}\left[\begin{array}{c}
(P+m+p: 1,1,1):(\alpha, 2) ;(1,1) ;(-p, 1) ; \\
-:(\beta, 2),\left(\frac{1}{2}, 1\right) ;(\lambda+1,1),(v+\lambda+1, \mu) ;(m+1,1)
\end{array} \quad \frac{w^{2}}{4 z},-\frac{q^{2}}{4 z}, \frac{x}{z}\right]\right. \\
& -\frac{w}{\sqrt{z}} \frac{\alpha}{\beta} \Gamma\left(P+p+m+\frac{1}{2}\right) \\
& \left.\times F_{0: 2 ; 2 ; 1}^{1: 1 ; 1 ; 1}\left[\begin{array}{cc}
\left(P+m+p+\frac{1}{2}: 1,1,1\right):(\alpha+1,2) ;(1,1) ;(-p, 1) ; & \\
-:(\beta+1,2),\left(\frac{3}{2}, 1\right) ;(\lambda+1,1),(v+\lambda+1, \mu) ;(m+1,1) ; & \frac{w^{2}}{4 z},-\frac{q^{2}}{4 z}, \frac{x}{z}
\end{array}\right]\right\} \\
& \left(q, w, z, Z, \mu, \alpha, \beta \in \mathfrak{R}^{+} ; \mathfrak{\Re}(\lambda)>-1, \Re(\nu)>0, \mathfrak{R}(\alpha)>-1, \Re(\beta)>-1, \Re(P)>0\right) \text {, }
\end{aligned}
$$

where $Z=z-s-t+\frac{x t}{s}$ and $P=\left(\frac{\eta+v+2 \lambda+1}{2}\right)$.

If we set $\beta=\alpha, \lambda=0$, and $\mu=1$ in equation (20), then it reduces to the know result given in $[18$, p. 62 , Eq. (4.1)].

On setting $q=0$ in equation (20), we obtain a (presumably) new relation between the generalized hypergeometric function and Kampé de Fériet function given by

$$
\begin{aligned}
& \left(\frac{z}{Z}\right)^{P}\left\{\Gamma(P)_{3} F_{3}\left[\begin{array}{ll}
P, \Delta(2 ; \alpha) ; & \\
\Delta(2 ; \beta), \frac{1}{2} ; & \frac{w^{2}}{4 Z}
\end{array}\right]\right. \\
& \left.\quad-\frac{\alpha w}{\beta \sqrt{Z}} \Gamma\left(P+\frac{1}{2}\right){ }_{3} F_{3}\left[\begin{array}{cc}
P+\frac{1}{2}, \Delta(2 ; \alpha+1) ; & \\
\Delta(2 ; \beta+1), \frac{3}{2} ; & \frac{w^{2}}{4 Z}
\end{array}\right]\right\} \\
& \quad=\sum_{m=-\infty}^{\infty} \sum_{p=0}^{\infty} \frac{\left(\frac{s}{z}\right)^{m}}{m !} \frac{\left(\frac{t}{z}\right)^{p}}{p !}
\end{aligned}
$$




$$
\begin{aligned}
& \times\left\{\Gamma(P+m+p) F_{0: 3 ; 1}^{1: 2 ; 1}\left[\begin{array}{c}
P+m+p: \Delta(2 ; \alpha) ;-p ; \\
-: \Delta(2 ; \beta), \frac{1}{2} ; m+1 ;
\end{array} \quad \frac{w^{2}}{4 z}, \frac{x}{z}\right]-\frac{w}{\sqrt{z}} \frac{\alpha}{\beta}\right. \\
& \left.\times \Gamma\left(P+p+m+\frac{1}{2}\right) F_{0: 3 ; 1}^{1: 2 ; 1}\left[\begin{array}{cc}
P+m+p+\frac{1}{2}: \Delta(2 ; \alpha+1) ;-p ; & \\
-: \Delta(2 ; \beta+1), \frac{3}{2} ; m+1 ; & \frac{w^{2}}{4 z}, \frac{x}{z}
\end{array}\right]\right\} \\
& \left(w, x, z, \alpha, \beta \in \mathfrak{R}^{+} ; \Re(P)>0\right) .
\end{aligned}
$$

For $\beta=\alpha, \lambda=0$, and $\mu=1$, equation (20) and equation (21) reduce to the known results (4.1) and (4.2), respectively, of Srivastava et al. [18, p. 62].

Further, on setting $w=0$ and replacing $P$ by $c$ in equation (21), we get

$$
\left(\frac{z}{Z}\right)^{c}=\sum_{m=-\infty}^{\infty} \sum_{p=0}^{\infty} \frac{\left(\frac{s}{z}\right)^{m}}{m !} \frac{\left(\frac{t}{z}\right)^{p}}{p !}(c)_{m+p 2} F_{1}\left[\begin{array}{cc}
c+m+p,-p ; & \\
m+1 ; & \frac{x}{z}
\end{array}\right] .
$$

Now, by using the definition of Jacobi polynomials $P_{n}^{(\alpha, \beta)}(x)$ (see [12, p. 254])

$$
P_{n}^{(\alpha, \beta)}(x)=\frac{(1+\alpha)_{n}}{n !}{ }_{2} F_{1}\left[\begin{array}{cc}
-n, \alpha+\beta+n+1 ; & \\
\alpha+1 ; & \frac{1-x}{z}
\end{array}\right],
$$

equation (22) reduces to the following known result of Pathan and Yasmeen [11, p. 242, Eq. (2.2)]:

$$
\begin{gathered}
\left(\frac{z}{Z}\right)^{c}=\sum_{m=-\infty}^{\infty} \sum_{p=0}^{\infty} \frac{\left(\frac{s}{z}\right)^{m}\left(\frac{t}{z}\right)^{p}}{(m+p) !}(c)_{m+p} P_{p}^{(m, c-1)}\left(\frac{z-2 x}{z}\right) \\
\left(x, z, Z \in \Re^{+} ; \Re(c)>0\right) .
\end{gathered}
$$

If we set $w=0$ in equation (20), then we obtain the following new generating relation between the generalized hypergeometric function and Kampé de Fériet function:

$$
\begin{aligned}
& \left(\frac{z}{Z}\right)^{P}{ }_{2} F_{\mu+1}\left[\begin{array}{cc}
P, 1 ; & -\frac{q^{2}}{4 Z \mu^{\mu}} \\
\lambda+1, \Delta(\mu ; \nu+\lambda+1) ; &
\end{array}\right] \\
& =\sum_{m=-\infty}^{\infty} \sum_{p=0}^{\infty} \frac{\left(\frac{s}{z}\right)^{m}}{m !} \frac{\left(\frac{t}{z}\right)^{p}}{p !}(P)_{m+p} \\
& \times F_{0: \mu+1 ; 1}^{1: 1 ; 1}\left[\begin{array}{cc}
P+m+p: 1 ;-p ; & \\
-: \lambda+1, \Delta(\mu ; \nu+\lambda+1) ; m+1 ; & -\frac{q^{2}}{4 z \mu^{\mu}}, \frac{x}{z}
\end{array}\right] \\
& \left(q, x, z, Z \in \mathfrak{R}^{+} ; \Re(\lambda)>-1, \Re(\nu)>0, \Re(P)>0\right) \text {. }
\end{aligned}
$$

For $\lambda=0$ and $\mu=1$, equation (25) reduces to the result (4.5) in [18, p. 63]. 
On setting $x=0$ in equation (25), we get the following generating function for the generalized hypergeometric function:

$$
\begin{aligned}
& \left(\frac{z}{Z_{1}}\right)^{P}{ }_{2} F_{\mu+1}\left[\begin{array}{cc}
P, 1 ; & -\frac{q^{2}}{4 Z_{1} \mu^{\mu}} \\
\lambda+1, \Delta(\mu ; \nu+\lambda+1) ; &
\end{array}\right] \\
& =\sum_{m=-\infty}^{\infty} \sum_{p=0}^{\infty} \frac{\left(\frac{s}{z}\right)^{m}}{m !} \frac{\left(\frac{t}{z}\right)^{p}}{p !} \\
& \times(P)_{m+p 2} F_{\mu+1}\left[\begin{array}{cc}
P+m+p: 1 ; & \\
\lambda+1, \Delta(\mu ; \nu+\lambda+1) ; & -\frac{q^{2}}{4 z \mu^{\mu}}
\end{array}\right] \\
& \left(q, z, Z_{1} \in \Re^{+} ; \Re(\lambda)>-1, \Re(v)>0, \Re(P)>0\right),
\end{aligned}
$$

where $P=\frac{\eta+v+\lambda+1}{2}$ and $Z_{1}=z-s-t$.

On putting $\lambda=0$ and $\mu=1$ in equation (26), we arrive at the following new generating function for the confluent hypergeometric function ${ }_{1} F_{1}$ :

$$
\begin{aligned}
& \left(\frac{z}{Z_{1}}\right)^{P_{1}}{ }_{1} F_{1}\left[P_{1} ; v+1 ;-\frac{q^{2}}{4 Z_{1}}\right] \\
& =\sum_{m=-\infty}^{\infty} \sum_{p=0}^{\infty} \frac{\left(\frac{s}{z}\right)^{m}}{m !} \frac{\left(\frac{t}{z}\right)^{p}}{p !}\left(P_{1}\right)_{m+p 1} F_{1}\left[P_{1}+m+p ; v+1 ;-\frac{q^{2}}{4 z}\right] \\
& \left(q, z, Z_{1} \in \mathfrak{R}^{+} ; \Re(v)>-1, \Re\left(P_{1}\right)>0\right),
\end{aligned}
$$

where $P_{1}=\frac{\eta+v+1}{2}$ and $Z_{1}=z-s-t$.

Further, on setting $\lambda=0, \mu=1, \eta=0$ and replacing $v$ by $2 v$ in equation (26), we get

$$
\begin{aligned}
& \left(\frac{z}{Z_{1}}\right)^{v+\frac{1}{2}}{ }_{1} F_{1}\left[v+\frac{1}{2} ; 2 v+1 ;-\frac{q^{2}}{4 Z_{1}}\right] \\
& \quad=\sum_{m=-\infty}^{\infty} \sum_{p=0}^{\infty} \frac{\left(\frac{s}{z}\right)^{m}}{m !} \frac{\left(\frac{t}{z}\right)^{p}}{p !}\left(v+\frac{1}{2}\right)_{m+p}{ }_{1} F_{1}\left[v+\frac{1}{2}+m+p ; 2 v+1 ;-\frac{q^{2}}{4 z}\right] .
\end{aligned}
$$

Now, using the following known result in equation (28) (see [16, p. 39, Eq. (21)]):

$$
{ }_{1} F_{1}\left[v+\frac{1}{2} ; 2 v+1 ;-2 x\right]=\Gamma(v+1) e^{-x}\left(\frac{x}{2}\right)^{-v} I_{v}(x),
$$

(where $I_{v}(x)$ is the modified Bessel function [16]), after a little simplification, we arrive at the following interesting result for the modified Bessel function:

$$
\begin{aligned}
I_{v}\left(\frac{q^{2}}{8 Z_{1}}\right)= & \frac{\left(\frac{Z_{1}}{z}\right)^{v+\frac{1}{2}} e^{\frac{q^{2}}{8 Z_{1}}}\left(\frac{q^{2}}{16 Z_{1}}\right)^{v}}{\Gamma(v+1)} \sum_{m=-\infty}^{\infty} \sum_{p=0}^{\infty} \frac{\left(\frac{s}{z}\right)^{m}}{m !} \frac{\left(\frac{t}{z}\right)^{p}}{p !} \\
& \times\left(v+\frac{1}{2}\right)_{m+p} F_{1} F_{1}\left[v+\frac{1}{2}+m+p ; 2 v+1 ;-\frac{q^{2}}{4 z}\right]
\end{aligned}
$$




$$
\left(q, z, Z_{1} \in \mathfrak{R}^{+} ; \Re\left(v+\frac{1}{2}\right)>0\right) .
$$

Further, using the following known result in equation (28) (see [16, p. 39, Eq. (20)]):

$$
{ }_{1} F_{1}\left[v+\frac{1}{2} ; 2 v+1 ; 2 i x\right]=\Gamma(v+1) e^{i x}\left(\frac{x}{2}\right)^{-v} J_{v}(x),
$$

(where $i=\sqrt{-1}$ and $J_{v}(x)$ is the Bessel function of the first kind [16]), we obtain a new result for Bessel function of the first kind:

$$
\begin{aligned}
J_{v}\left(\frac{i q^{2}}{8 Z_{1}}\right)= & \frac{\left(\frac{Z_{1}}{z}\right)^{v+\frac{1}{2}} e^{\frac{q^{2}}{8 Z_{1}}}\left(\frac{i q^{2}}{16 Z_{1}}\right)^{v}}{\Gamma(v+1)} \sum_{m=-\infty}^{\infty} \sum_{p=0}^{\infty} \frac{\left(\frac{s}{z}\right)^{m}}{m !} \frac{\left(\frac{t}{z}\right)^{p}}{p !} \\
& \times\left(v+\frac{1}{2}\right)_{m+p} F_{1}\left[v+\frac{1}{2}+m+p ; 2 v+1 ;-\frac{q^{2}}{4 z}\right] \\
& \left(q, z, Z_{1} \in \Re^{+} ; \Re\left(v+\frac{1}{2}\right)>0\right) .
\end{aligned}
$$

If we expand ${ }_{1} F_{1}$ in its defining series in equation (30) and equation (32), respectively, and arrange the resulting expressions into Kampé de Fériet function, then we get the following interesting relations between Bessel's functions of the first kind and Kampé de Fériet function:

$$
\begin{aligned}
I_{v}\left(\frac{q^{2}}{8 Z_{1}}\right)= & \frac{\left(\frac{Z_{1}}{z}\right)^{v+\frac{1}{2}} e^{\frac{q^{2}}{8 Z_{1}}\left(\frac{q^{2}}{16 Z_{1}}\right)^{v}}}{\Gamma(v+1)} \sum_{m=-\infty}^{\infty} \frac{\left(\frac{s}{z}\right)^{m}}{m !}\left(v+\frac{1}{2}\right)_{m} \\
& \left.\times F_{0: 0 ; 1}^{1: 0 ; 0}\left[\begin{array}{c}
v+\frac{1}{2}+m:-;-; \\
-:-; 2 v+1) ;
\end{array}\right] \frac{\frac{t}{z},-\frac{q^{2}}{4 z}}{-}\right]
\end{aligned}
$$

and

$$
\begin{aligned}
J_{v}\left(\frac{i q^{2}}{8 Z_{1}}\right)= & \frac{\left(\frac{Z_{1}}{z}\right)^{v+\frac{1}{2}} e^{\frac{q^{2}}{8 Z_{1}}}\left(\frac{i q^{2}}{16 Z_{1}}\right)^{v}}{\Gamma(v+1)} \sum_{m=-\infty}^{\infty} \frac{\left(\frac{s}{z}\right)^{m}}{m !}\left(v+\frac{1}{2}\right)_{m} \\
& \times F_{0: 0 ; 1}^{1: 0 ; 0}\left[\begin{array}{c}
v+\frac{1}{2}+m:-;-; \\
-:-; 2 v+1 ;
\end{array}\right] \\
& \left(q, t, z, Z_{1} \in \Re^{+} ; \Re\left(v+\frac{q^{2}}{4 z}\right)\right) .
\end{aligned}
$$

On setting $\lambda=0, \mu=1$, replacing $\eta$ by $2 \eta$ and $v$ by $2 v$ in equation (26), we get

$$
\begin{gathered}
\left(\frac{z}{Z_{1}}\right)^{\eta+v+\frac{1}{2}}{ }_{1} F_{1}\left[\eta+v+\frac{1}{2} ; 2 v+1 ;-\frac{q^{2}}{4 Z_{1}}\right] \\
=\sum_{m=-\infty}^{\infty} \sum_{p=0}^{\infty} \frac{\left(\frac{s}{z}\right)^{m}}{m !} \frac{\left(\frac{t}{z}\right)^{p}}{p !}
\end{gathered}
$$




$$
\times\left(\eta+v+\frac{1}{2}\right)_{m+p}{ }_{1} F_{1}\left[\eta+v+\frac{1}{2}+m+p ; 2 v+1 ;-\frac{q^{2}}{4 z}\right]
$$

Now, by using the under mentioned definition of Whittaker function $M_{k, \mu}(x)$ (see [16, p. 39, Eq. (23)]) in equation (35)

$$
M_{k, \mu}(x)=x^{\mu+\frac{1}{2}} e^{\frac{x}{2}}{ }_{1} F_{1}\left[\mu+k+\frac{1}{2} ; 2 \mu+1 ;-x\right],
$$

we arrive at the following (presumably) new result for the Whittaker function of the first kind:

$$
\begin{aligned}
& M_{\eta, v}\left(\frac{q^{2}}{4 Z_{1}}\right)=\left(\frac{Z_{1}}{z}\right)^{\eta} e^{\frac{q^{2}}{8}\left(\frac{1}{Z_{1}}-\frac{1}{z}\right)} \\
& \times \sum_{m=-\infty}^{\infty} \sum_{p=0}^{\infty} \frac{\left(\frac{s}{z}\right)^{m}}{m !} \frac{\left(\frac{t}{z}\right)^{p}}{p !}\left(\eta+v+\frac{1}{2}\right)_{m+p} M_{\eta+m+p, v}\left(\frac{q^{2}}{4 z}\right) \\
&\left(q, z, Z_{1} \in \Re^{+} ; \Re\left(\eta+v+\frac{1}{2}\right)>0\right) .
\end{aligned}
$$

Further, on setting $\lambda=0, \mu=1, v=\frac{1}{2}$, and $\eta=\frac{1}{2}$ in equation (26), and then by using the definition of error function

$$
{ }_{1} F_{1}\left(\frac{1}{2} ; \frac{3}{2} ;-x^{2}\right)=\frac{\sqrt{\pi}}{2 x} \operatorname{erf}(x)
$$

we arrive at

$$
\operatorname{erf}\left(\frac{q}{2 \sqrt{Z_{1}}}\right)=\frac{1}{\sqrt{(} \pi z)} \sum_{m=-\infty}^{\infty} \sum_{p=0}^{\infty} \frac{\left(\frac{s}{z}\right)^{m}}{m !} \frac{\left(\frac{t}{z}\right)^{p}}{p !}\left(\frac{1}{2}\right)_{m+p}{ }_{1} F_{1}\left[\frac{1}{2}+m+p ; \frac{3}{2} ;-\frac{q^{2}}{4 z}\right] .
$$

In equation (39), on expanding ${ }_{1} F_{1}$ in a series form and arranging the resulting expression into Kampé de Fériet function, we get

$$
\begin{aligned}
& \operatorname{erf}\left(\frac{q}{2 \sqrt{Z}_{1}}\right)=\frac{1}{\sqrt{ }(\pi z)} \sum_{m=-\infty}^{\infty} \frac{\left(\frac{s}{z}\right)^{m}}{m !}\left(\frac{1}{2}\right)_{m} F_{0: 0 ; 1}^{1: 0 ; 0}\left[\begin{array}{cc}
m+\frac{1}{2}:-;-; & \frac{t}{z},-\frac{q^{2}}{4 z} \\
-:-; \frac{3}{2} ; &
\end{array}\right] \\
& \left(q, t, z, Z_{1} \in \Re^{+}\right) .
\end{aligned}
$$

\section{Recurrence relations}

In this section, we present the following recurrence relations for our introduced extended Voigt function $\Lambda_{\eta, v, \lambda}^{\mu, \alpha}$ :

$$
\begin{aligned}
& (\beta-\alpha) \Lambda_{\eta, v, \lambda}^{\mu, \alpha-1, \beta}+(2 \alpha-\beta) \Lambda_{\eta, v, \lambda}^{\mu, \alpha, \beta}-y \Lambda_{\eta+1, v, \lambda}^{\mu, \alpha, \beta}-\alpha \Lambda_{\eta, v, \lambda}^{\mu, \alpha+1, \beta}=0 \\
& \beta(\beta-1) \Lambda_{\eta, v, \lambda}^{\mu, \alpha, \beta-1}-\beta(\beta-1) \Lambda_{\eta, v, \lambda}^{\mu, \alpha, \beta}+\beta y \Lambda_{\eta+1, v, \lambda}^{\mu, \alpha, \beta}-y(\beta-\alpha) \Lambda_{\eta+1, v, \lambda}^{\mu, \alpha, \beta+1}=0 \\
& (1+\alpha-\beta) \Lambda_{\eta, v, \lambda}^{\mu, \alpha, \beta}-\alpha \Lambda_{\eta, v, \lambda}^{\mu, \alpha+1, \beta}+(\beta-1) \Lambda_{\eta, v, \lambda}^{\mu, \alpha, \beta-1}=0 \\
& \beta \Lambda_{\eta, v, \lambda}^{\mu, \alpha, \beta}-\beta \Lambda_{\eta, v, \lambda}^{\mu, \alpha+1, \beta}+y \Lambda_{\eta+1, v, \lambda}^{\mu, \alpha, \beta+1}=0
\end{aligned}
$$




$$
\begin{aligned}
& \alpha \beta \Lambda_{\eta, v, \lambda}^{\mu, \alpha, \beta}-\beta y \Lambda_{\eta+1, v, \lambda}^{\mu, \alpha, \beta}+y(\beta-\alpha) \Lambda_{\eta+1, \nu, \lambda}^{\mu, \alpha, \beta+1}-\alpha \beta \Lambda_{\eta, v, \lambda}^{\mu, \alpha+1, \beta}=0 ; \\
& (\alpha-1) \Lambda_{\eta, \nu, \lambda}^{\mu, \alpha, \beta}-y \Lambda_{\eta+1, \nu, \lambda}^{\mu, \alpha, \beta}+(\beta-\alpha) \Lambda_{\eta, \nu, \lambda}^{\mu, \alpha-1, \beta}-(\beta-1) \Lambda_{\eta, \nu, \lambda}^{\mu, \alpha, \beta-1}=0 .
\end{aligned}
$$

Here, we omit the variables.

Proof We have the following recurrence relation of the confluent hypergeometric function ${ }_{1} F_{1}$ (see [13, p. 19]):

$$
(b-a)_{1} F_{1}(a-1 ; b ; x)+(2 a-b){ }_{1} F_{1}(a ; b ; x)+x_{1} F_{1}(a ; b ; x)-a_{1} F_{1}(a+1 ; b ; x)=0 .
$$

By using the above relation, we can easily arrive at

$$
\begin{aligned}
& (\beta-\alpha) \sqrt{\frac{x}{2}} \int_{0}^{\infty} t^{\eta} e^{-z t^{2}}{ }_{1} F_{1}(\alpha-1 ; \beta ;-y t) J_{v, \lambda}^{\mu}(x t) d t \\
& +(2 \alpha-\beta) \sqrt{\frac{x}{2}} \int_{0}^{\infty} t^{\eta} e^{-z t^{2}}{ }_{1} F_{1}(\alpha ; \beta ;-y t) J_{v, \lambda}^{\mu}(x t) d t \\
& -y \sqrt{\frac{x}{2}} \int_{0}^{\infty} t^{\eta+1} e^{-z t^{2}}{ }_{1} F_{1}(\alpha ; \beta ;-y t) J_{v, \lambda}^{\mu}(x t) d t \\
& -\alpha \sqrt{\frac{x}{2}} \int_{0}^{\infty} t^{\eta} e^{-z t^{2}}{ }_{1} F_{1}(\alpha+1 ; \beta ;-y t) J_{v, \lambda}^{\mu}(x t) d t=0 .
\end{aligned}
$$

Note here, on applying definition (3) in the above expression yields the first recurrence relation (41).

A similar argument can establish the other formulas given in equations (42)-(46), respectively, by using the following recurrence relations of ${ }_{1} F_{1}$ (see [13, p. 19]):

$$
\begin{aligned}
& b(b-1){ }_{1} F_{1}(a ; b-1 ; x)-b(b-1){ }_{1} F_{1}(a ; b ; x)-b x_{1} F_{1}(a ; b ; x) \\
& \quad+(b-a) x_{1} F_{1}(a ; b+1 ; x)=0 ; \\
& (1+a-b){ }_{1} F_{1}(a ; b ; x)-a_{1} F_{1}(a+1 ; b ; x)+(b-1)_{1} F_{1}(a ; b-1 ; x)=0 ; \\
& b_{1} F_{1}(a ; b ; x)-b_{1} F_{1}(a-1 ; b ; x)-x_{1} F_{1}(a ; b+1 ; x)=0 ; \\
& a b_{1} F_{1}(a ; b ; x)+b x_{1} F_{1}(a ; b ; x)-(b-a) x_{1} F_{1}(a ; b+1 ; x)-a b_{1} F_{1}(a+1 ; b ; x)=0 ; \\
& (a-1)_{1} F_{1}(a ; b ; x)+x_{1} F_{1}(a ; b ; x)+(b-a)_{1} F_{1}(a-1 ; b ; x) \\
& \quad-(b-1){ }_{1} F_{1}(a ; b-1 ; x)=0 .
\end{aligned}
$$

\section{Some recurrence type connection formulas}

Here, we present some recurrence type connection formulas for our extended Voigt function $\Lambda_{\eta, v, \lambda}^{\mu, \alpha, \beta}$ and the Voigt function $\Omega_{\eta, v, \lambda}^{\mu}$ defined by Srivastava et al. [18] as follows:

$$
\begin{aligned}
& \alpha \Omega_{\eta, v, \lambda}^{\mu}-y \Omega_{\eta+1, v, \lambda}^{\mu}-\alpha \Lambda_{\eta, v, \lambda}^{\mu, \alpha, \alpha+1}=0 \\
& \alpha(\alpha-1) \Lambda_{\eta, v, \lambda}^{\mu, \alpha, \alpha-1}-\alpha(\alpha-1) \Omega_{\eta, v, \lambda}^{\mu}+\alpha y \Omega_{\eta+1, v, \lambda}^{\mu}=0 \\
& \Omega_{\eta+1, v, \lambda}^{\mu}-\alpha \Lambda_{\eta, v, \lambda}^{\mu, \alpha+1, \beta}+(\alpha-1) \Lambda_{\eta, v, \lambda}^{\mu, \alpha, \alpha-1}=0
\end{aligned}
$$




$$
\begin{aligned}
& \alpha \Omega_{\eta, v, \lambda}^{\mu}-\beta \Lambda_{\eta, v, \lambda}^{\mu, \alpha-1, \alpha}+y \Lambda_{\eta+1, v, \lambda}^{\mu, \alpha, \alpha+1}=0 ; \\
& \alpha \Omega_{\eta, v, \lambda}^{\mu}-y \Omega_{\eta+1, v, \lambda}^{\mu}-\alpha \Lambda_{\eta, v, \lambda}^{\mu, \alpha+1, \beta}=0 ; \\
& (\alpha-1) \Omega_{\eta, v, \lambda}^{\mu}-y \Omega_{\eta+1, v, \lambda}^{\mu}-(\alpha-1) \Lambda_{\eta, v, \lambda}^{\mu, \alpha, \alpha-1}=0 ;
\end{aligned}
$$

Here, we omit the variables.

Proof On taking $\beta=\alpha$ in equation (48) and then using the definition of Voigt functions given by equation (1) and equation (3), respectively, we get our first formula (54). The other formulas can be established (in a similar way) by taking $\beta=\alpha$ in the integral representation of relations (42), (43), (44), (45), and (46), respectively.

\section{Acknowledgements}

Not applicable.

\section{Funding}

The author T.A. would like to thank Prince Sultan University for the support through the research group Nonlinear Analysis Methods in Applied Mathematics (NAMAM) group number RG-DES-2017-01-17.

\section{Availability of data and materials}

Not applicable.

\section{Competing interests}

The authors declare that they have no competing interests.

\section{Authors' contributions}

The authors contributed equally and significantly in writing this paper. All authors read and approved the final manuscript.

\section{Author details}

${ }^{1}$ Department of Applied Mathematics, Faculty of Engineering and Technology, Aligarh Muslim University, Aligarh, India. ${ }^{2}$ Department of Mathematics, Integral University Campus, Shahjahanpur, India. ${ }^{3}$ Department of Mathematics and Natural Sciences, Prince Mohammad Bin Fahd University, Al Khobar, Kingdom of Saudi Arabia. ${ }^{4}$ Department of Mathematics and General Sciences, Prince Sultan University, Riyadh, Kingdom of Saudi Arabia. ${ }^{5}$ Department of Medical Research, China Medical University, Taichung, Taiwan. ${ }^{6}$ Department of Computer Science and Information Engineering, Asia University, Taichung, Taiwan. ${ }^{7}$ Department of Mathematics, College of Arts and Sciences, Prince Sattam bin Abdulaziz University, Wadi Aldawasir, Kingdom of Saudi Arabia.

\section{Publisher's Note}

Springer Nature remains neutral with regard to jurisdictional claims in published maps and institutional affiliations.

Received: 20 February 2020 Accepted: 27 April 2020 Published online: 24 May 2020

\section{References}

1. Erdélyi, A., Magnus, W., Oberhettinger, F., Tricomi, F.G.: Table of Integral Transforms. Vol. I. MaGraw-Hill, New York (1954)

2. Goyal, S.P., Mukherjee, R.: Generalizations of the Voigt functions through generalized Lauricella function. Ganita Sandesh 13(1), 31-41 (1999)

3. Gupta, K., Gupta, A.: On the study of unified representations of the generalized Voigt functions. Palestine J. Math. 2(1) $32-37(2013)$

4. Gupta, K.C., Goyal, S.P., Mukherjee, R.: Some results on generalized Voigt functions. ANZIAM J. 44, 299-303 (2002)

5. Khan, N.U., Kamarujjama, M., Ghayasuddin, M.: A generalization of Voigt function involving generalized Whittaker and Bessel functions. Palestine J. Math. 4(2), 313-318 (2015)

6. Klusch, D.: Astrophysical spectroscopy and neutron reactions, integral transforms and Voigt functions. Astrophys. Space Sci. 175, 229-240 (1991)

7. Pathak, R.S.: Certain convergence theorems and asymptotic properties of a generalization of Lommel and Maitland transform. Proc. Natl. Acad. Sci. India Sect. A 36, 81-86 (1996)

8. Pathan, M.A., Garg, M., Mittal, S.: On unified presentations of the multivariable Voigt functions. East-West J. Math. 8(1), 49-59 (2006)

9. Pathan, M.A., Gupta, K., Agrawal, V.: Summation formulae involving Voigt functions and generalized hypergeometric functions. Scientia, Ser. A, Math. Sci. 19,37-44 (2010)

10. Pathan, M.A., Shahwan, M.J.S.: New representation of the Voigt functions. Demonstr. Math. 39, 1-4 (2006)

11. Pathan, M.A., Yasmeen: On partly bilateral and partly unilateral generating functions. J. Aust. Math. Soc. Ser. B 28 240-245 (1986) 
12. Rainville, E.D.: Special Functions. Macmillan Co., New York (1960)

13. Slater, L.J.: Confluent Hypergeometric Functions. Cambridge University Press, Cambridge (1960)

14. Srivastava, H.M., Bin-Saad, M.G., Pathan, M.A.: A new theorem on multidimensional generating relations and its applications. Proc. Jangjeon Math. Soc. 10(1), 7-22 (2007)

15. Srivastava, H.M., Chen, M.-P.: Some unified presentations of the Voigt functions. Astrophys. Space Sci. 192, 63-74 (1992)

16. Srivastava, H.M., Manocha, H.L.: A Treatise on Generating Functions. Halsted Press, New York (1984)

17. Srivastava, H.M., Miller, E.A.: A unified presentation of the Voigt functions. Astrophys. Space Sci. 135, 111-118 (1987)

18. Srivastava, H.M., Pathan, M.A., Kamarujjama, M.: Some unified presentations of the generalized Voigt functions. Commun. Appl. Anal. 2, 49-64 (1998)

Submit your manuscript to a SpringerOpen ${ }^{\circ}$ journal and benefit from:

- Convenient online submission

- Rigorous peer review

- Open access: articles freely available online

- High visibility within the field

- Retaining the copyright to your article

Submit your next manuscript at $\boldsymbol{~ s p r i n g e r o p e n . c o m ~}$ 